\title{
Association of osteopenia and osteoporosis with higher rates of pseudarthrosis and revision surgery in adult patients undergoing single-level lumbar fusion
}

\author{
Syed I. Khalid, MD, ${ }^{1}$ Ravi S. Nunna, MD, ${ }^{2}$ Samantha Maasarani, MPH, ${ }^{3}$ Erika Belmont, BA, ${ }^{3}$ \\ Palvasha Deme, BA, ${ }^{4}$ Sai Chilakapati, MS,${ }^{4}$ Cody Eldridge, BS, ${ }^{4}$ Ravinderjit Singh, BS, ${ }^{4}$ \\ Carlos A. Bagley, MD, ${ }^{4}$ and Owoicho Adogwa, MD, MPH ${ }^{4}$ \\ ${ }^{1}$ Department of Surgery, Rush University Medical Center, Chicago; 2Department of Neurosurgery, University of Illinois at \\ Chicago; ${ }^{3}$ Chicago Medical School, Rosalind Franklin University, North Chicago, Illinois; and 4 Department of Neurosurgery, \\ University of Texas Southwestern Medical School, Dallas, Texas
}

\begin{abstract}
OBJECTIVE Patients with osteopenia or osteoporosis who require surgery for symptomatic degenerative spondylolisthesis may have higher rates of postoperative pseudarthrosis and need for revision surgery than patients with normal bone mineral densities (BMDs). To this end, the authors compared rates of postoperative pseudarthrosis and need for revision surgery following single-level lumbar fusion in patients with normal BMD with those in patients with osteopenia or osteoporosis. The secondary outcome was to investigate the effects of pretreatment with medications that prevent bone loss (e.g., teriparatide, bisphosphonates, and denosumab) on these adverse outcomes in this patient cohort.

METHODS Patients undergoing single-level lumbar fusion between 2007 and 2017 were identified. Based on 1:1 propensity matching for baseline demographic characteristics and comorbidities, 3 patient groups were created: osteopenia $(n=1723,33.3 \%)$, osteoporosis $(n=1723,33.3 \%)$, and normal BMD $(n=1723,33.3 \%)$. The rates of postoperative pseudarthrosis and revision surgery were compared between groups.

RESULTS The matched populations analyzed in this study included a total of 5169 patients in 3 groups balanced at baseline, with equal numbers $(n=1723,33.3 \%)$ in each group: patients with a history of osteopenia, those with a history of osteoporosis, and a control group of patients with no history of osteopenia or osteoporosis and with normal BMD. A total of 597 complications were recorded within a 2-year follow-up period, with pseudarthrosis $(n=321,6.2 \%)$ being slightly more common than revision surgery $(n=276,5.3 \%)$. The odds of pseudarthrosis and revision surgery in patients with osteopenia were almost 2-fold (OR 1.7, 95\% Cl 1.26-2.30) and 3-fold (OR 2.73, 95\% Cl 1.89-3.94) higher, respectively, than those in patients in the control group. Similarly, the odds of pseudarthrosis and revision surgery in patients with osteoporosis were almost 2-fold (OR 1.92, 95\% Cl 1.43-2.59) and > 3-fold (OR 3.25, 95\% Cl 2.27-4.65) higher, respectively, than those in patients in the control group. Pretreatment with medications to prevent bone loss prior to surgery was associated with lower pseudarthrosis and revision surgery rates, although the differences did not reach statistical significance.
\end{abstract}

CONCLUSIONS Postoperative pseudarthrosis and revision surgery rates following single-level lumbar spinal fusion are significantly higher in patients with osteopenia and osteoporosis than in patients with normal BMD. Pretreatment with medications to prevent bone loss prior to surgery decreased these complication rates, although the observed differences did not reach statistical significance.

https://thejns.org/doi/abs/10.3171/2020.5.FOCUS20289

KEYWORDS osteoporosis; osteopenia; degenerative lumbar spondylolisthesis; teriparatide; bisphosphonates; denosumab; aging spine

A $\mathrm{s}$ the number of older adults in the United States continues to rise, there will be an associated increase in age-related diseases, such as degenerative disorders of the spine. Low-back pain (LBP) secondary to degenerative lumbar stenosis and spondylolisthesis are common causes of functional impairment and inability to perform essential activities of daily living. While the majority of patients with symptomatic LBP are managed nonoperatively, in a subset of patients nonoperative management is unsuccessful and surgery may be required.

ABBREVIATIONS BMD = bone mineral density; CPT = Current Procedural Terminology; DEXA = dual-energy $\mathrm{x}$-ray absorptiometry; ECI = Elixhauser Comorbidity Index. SUBMITTED April 3, 2020. ACCEPTED May 12, 2020.

INCLUDE WHEN CITING DOI: 10.3171/2020.5.FOCUS20289. 
When indicated, lumbar spinal fusion leads to superior improvements in pain, disability, and quality of life, as well as increased activity levels because of the absence of mechanical back pain., ${ }^{1,2}$

Osteopenia and osteoporosis are common among older adults with degenerative disorders of the spine. ${ }^{3,4}$ In the United States alone, over 10 million people are estimated to have osteoporosis. ${ }^{5}$ An additional 34 million older adults are estimated to have osteopenia, which puts them at increased risk for developing osteoporosis. ${ }^{6,7}$ Patients with osteoporosis or osteopenia undergoing lumbar fusions have an elevated risk of postoperative complications that may require revision surgery-including pedicle screw loosening, nonunion, proximal junctional kyphosis, and adjacent segment fractures. ${ }^{5,8-11}$ However, there is a paucity of high-quality population-level analysis documenting the prevalence of osteoporosis and osteopenia in patients with degenerative disorders of the spine requiring spinal fusion, and hence the absolute risk of pseudarthrosis and revision surgery remains unclear.

We hypothesized that patients with osteoporosis and osteopenia undergoing single-level lumbar fusion would have higher rates of pseudarthrosis and need for revision surgery. To this end, the primary aim of this study was to investigate the effects of osteopenia or osteoporosis on the rate of pseudarthrosis and need for revision surgery following single-level lumbar spinal fusion. The secondary aim was to investigate the effects of pretreatment with medications that prevent bone loss on these outcomes in patients with osteoporosis or osteopenia.

\section{Methods \\ Data Source}

This study followed the STROBE (Strengthening the Reporting of Observational Studies in Epidemiology) reporting guidelines. Longitudinal analytical files containing all medical, drug, and laboratory claims of 22 million patients insured by Humana, an American health insurance company, between January 1, 2007, and March 31, 2017, were utilized in this cohort study. Patients were identified as having undergone single-level lumbar fusion based on Current Procedural Terminology (CPT) and ICD-9 and -10 procedure codes, as shown in Supplemental Table 1. Patients within this population who had a history of osteopenia or osteoporosis were identified based on ICD-9 and -10 diagnosis codes, also shown in Supplemental Table 1 . The study was approved by the Institutional Review Board with a waiver of patient informed consent, as the nature of this analysis posed minimal risk to participating individuals, and the data were presented in aggregate to minimize any risks of loss of confidentiality of medical data.

\section{Comorbidities and Indications for Surgery}

Demographic data for aggregate records included age and sex. Postmenopausal status was identified as female patients aged $\geq 50$ years. ICD- 9 and -10 diagnosis codes were used to identify comorbidities and indications for surgery, as listed in Supplemental Table 2. Comorbidities included were as follows: $\mathrm{BMI} \geq 30 \mathrm{~kg} / \mathrm{m}^{2}$, current or former smoking history, diabetes mellitus, and hypertension. Indications for surgery were classified as either spondylosis, spondylolisthesis, or intervertebral disc disease.

\section{Study Outcomes}

The primary aim of this study was to assess the effects of osteopenia and osteoporosis on the odds of developing pseudarthrosis or need for revision surgery within 2 years postoperatively in patients undergoing single-level lumbar fusion procedures. Pseudarthrosis and need for revision surgery were identified using ICD-9 and -10 diagnosis and procedure codes as seen in Supplemental Table 3.

The secondary aim of this study was to assess the mitigation of osteoporotic and osteopenic risk by means of preoperative medical treatment with medications (teriparatide, bisphosphonates, and denosumab) that prevent bone loss. These variables were assessed by measuring the odds of developing pseudarthrosis and the need for revision surgery within 2 years postoperatively in osteopenic and osteoporotic patients with and without histories of taking these medications within 30 days preoperatively. These medications were identified by utilizing specific drug category bucket names as shown in Supplemental Table 4.

\section{Statistical Analysis}

Descriptive statistics were calculated for age, sex, and comorbidities and compared between the 3 groups: patients with a history of osteopenia, those with a history of osteoporosis, and control patients with no history of osteopenia or osteoporosis. Using propensity matching, patient populations were exactly matched in a 1:1 fashion based on their age, sex, Elixhauser Comorbidity Index (ECI) score, BMI of $30 \mathrm{~kg} / \mathrm{m}^{2}$ or more, postmenopausal status, current or former smoking history, history of diabetes mellitus, and history of hypertension. Chi-square tests were then calculated to compare categorical variables: age ranges, sex, and comorbidity status (BMI of $30 \mathrm{~kg} / \mathrm{m}^{2}$ or more, postmenopausal status, smoking, diabetes mellitus, and hypertension). ANOVA was used for the quantitative variable ECI score. Odds ratios were calculated to compare pseudarthrosis and need for revision surgery events based on whether or not the patient had a history of osteopenia, osteoporosis, or neither. Kaplan-Meier survival analyses of pseudarthrosis and revision surgery for patients with a history of osteopenia, osteoporosis, or neither were plotted for 2 years following surgery.

\section{Results}

\section{Descriptive Characteristics}

Single-level lumbar fusion patients were identified based on CPT and ICD-9 and -10 procedural codes between 2007 and $2017(n=72,899)$. Following exclusion of patients with ages younger than 20 years or older than 80 years $(n=5825)$ and those with an inactive or unknown Humana insurance record for the 2-year follow-up period $(n=39,315), 27,759$ eligible patients were identified, with 3272 of these having a history of osteopenia, 2496 a history of osteoporosis, and 21,991 no history of osteopenia 


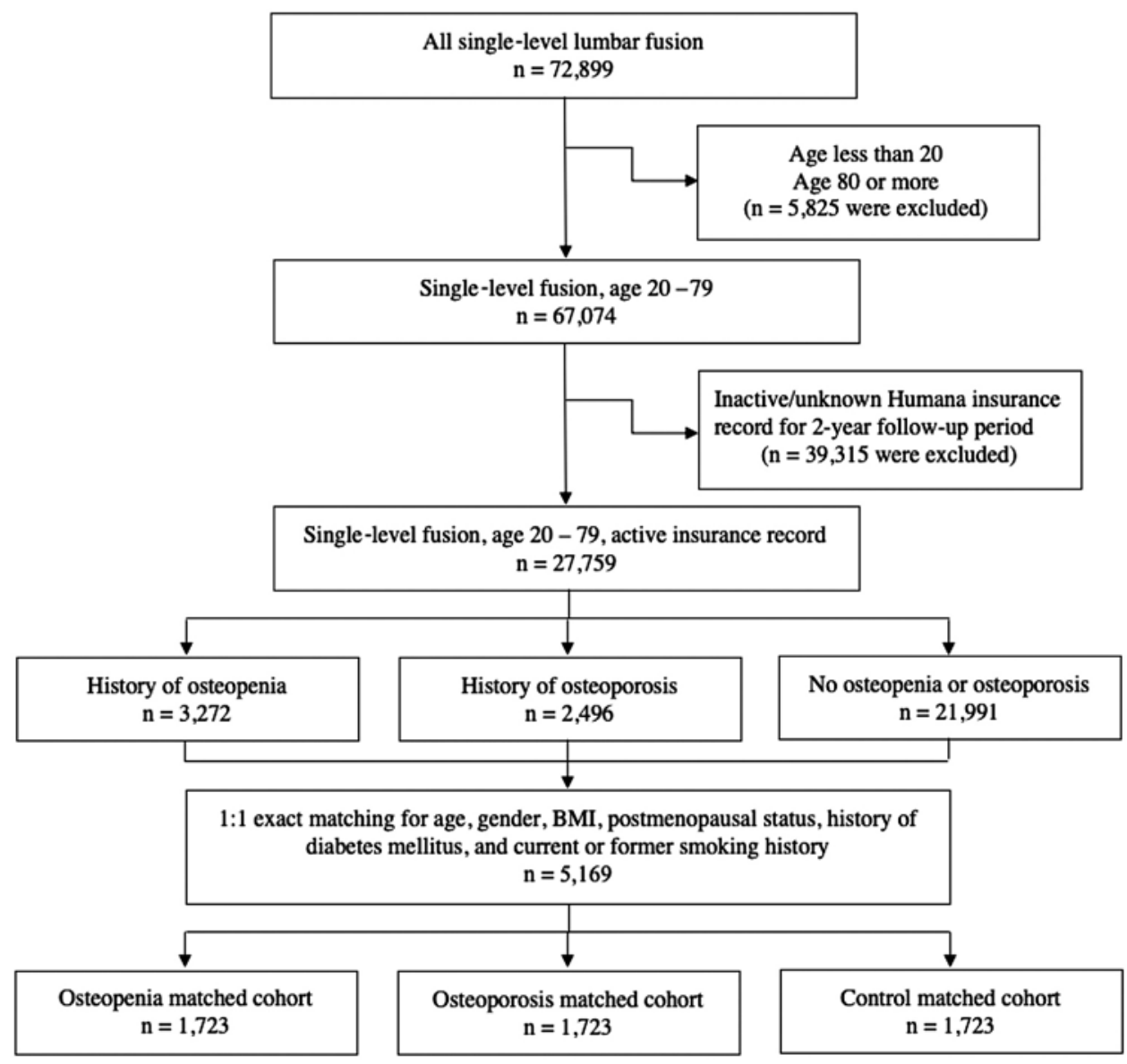

FIG. 1. Patient selection flowchart.

or osteoporosis, as seen in Fig. 1. Indications for surgery have been summarized in Supplemental Table 5. Patient characteristics and comorbidity status for the unmatched population can be seen in Supplemental Table 6 .

The propensity-matched population analyzed in this study included 5169 patients, who were equally represented by patients with a history of osteopenia $(n=1723$, $33.3 \%)$, a history of osteoporosis $(\mathrm{n}=1723,33.3 \%)$, or no history of osteopenia or osteoporosis $(\mathrm{n}=1723,33.3 \%)$. Patient demographic characteristics and comorbidity status for the matched population can be seen in Table 1. All 3 patient groups were balanced at baseline with respect to sex, age, BMI, postmenopausal status, smoking status, history of diabetes mellitus, and history of hypertension.

The mean ECI \pm SD score for each cohort was $9.81 \pm$ 4.23. The osteopenia and osteoporosis groups were subclassified depending on their preoperative medication history status, as seen in Tables 2 and 3. A majority of patients with osteopenia were not taking any medications (90.02\%); of those taking medications, bisphosphonates (n $=170,98.84 \%)$ and teriparatide $(\mathrm{n}=2,1.16 \%)$ were most common. Similarly, only a minority of patients with a diagnosis of osteoporosis were taking medications (27.68\%), with bisphosphonates $(\mathrm{n}=453,94.97 \%)$ being more commonly used than teriparatide $(\mathrm{n}=18,3.77 \%)$ and denosumab $(n=6,1.26 \%)$.

\section{Role of Decreased Bone Densities in Postoperative Outcomes}

A total of 597 complications occurred within our 2-year follow-up period, with pseudarthrosis $(\mathrm{n}=321,6.2 \%)$ being slightly more common than revision surgery $(n=276$, $5.3 \%$ ), as shown in Table 1. More specifically, pseudarthrosis was more common in patients with a history of osteoporosis $(\mathrm{n}=132,7.7 \%)$ than in those with osteopenia $(\mathrm{n}=118,6.8 \%)$ and the control cohort $(\mathrm{n}=71,4.1 \%)$, and this difference trended toward statistical significance (logrank $\mathrm{p}=0.08$; Fig. 2). The rates of revision surgery were statistically significantly different $(\log -$ rank $\mathrm{p}=0.02$; Fig. 2 ) and were more common in the osteoporosis cohort ( $\mathrm{n}=$ $127,7.4 \%)$ than the osteopenia $(\mathrm{n}=108,6.3 \%)$ and control ( $\mathrm{n}=41,2.4 \%)$ cohorts.

Furthermore, the odds of pseudarthrosis and revision surgery in patients with osteopenia were almost 2-fold (OR 1.7, 95\% CI 1.26-2.30) and 3-fold (OR 2.73, 95\% CI 1.89-3.94) higher, respectively, than those in patients in the control group, who all had normal bone mineral density (BMD; Table 4). Similarly, the odds of pseudarthrosis and revision surgery in patients with osteoporosis were almost 2-fold (OR 1.92, 95\% CI 1.43-2.59) and over 3-fold (OR 3.25, 95\% CI 2.27-4.65) higher, respectively, than those in patients in the control group, all of whom had normal BMD (Table 4). 
TABLE 1. Descriptive characteristics for matched patients undergoing single-level lumbar fusion

\begin{tabular}{|c|c|c|c|c|c|}
\hline & Total $(n=5169)$ & \multicolumn{3}{|c|}{ Control, Osteopenia, \& Osteoporosis Groups ( $n=1723$ /group) } & $\mathrm{p}$ Value \\
\hline Age, yrs & & & & & 0.99 \\
\hline $40-44$ & $18(0.3 \%)$ & & $6(0.3 \%)$ & & \\
\hline $45-49$ & $45(0.9 \%)$ & & $15(0.9 \%)$ & & \\
\hline $50-54$ & $216(4.2 \%)$ & & $72(4.2 \%)$ & & \\
\hline $55-59$ & $411(8.0 \%)$ & & $137(8.0 \%)$ & & \\
\hline $60-64$ & $594(11.5 \%)$ & & $198(11.5 \%)$ & & \\
\hline $65-69$ & $1905(36.9 \%)$ & & $635(36.9 \%)$ & & \\
\hline $70-74$ & $1431(27.7 \%)$ & & $477(27.7 \%)$ & & \\
\hline $75-79$ & $549(10.6 \%)$ & & $183(10.6 \%)$ & & \\
\hline Sex & & & & & 0.99 \\
\hline Female & $4413(85.4 \%)$ & & $1471(85.4 \%)$ & & \\
\hline Male & $756(14.6 \%)$ & & $252(14.6 \%)$ & & \\
\hline Comorbidities & & & & & 0.99 \\
\hline $\mathrm{BMI} \geq 30 \mathrm{~kg} / \mathrm{m}^{2}$ & $723(14.0 \%)$ & & $241(14.0 \%)$ & & \\
\hline Postmenopausal & $4383(84.8 \%)$ & & $1461(84.8 \%)$ & & \\
\hline Smoking & $174(3.4 \%)$ & & $58(3.4 \%)$ & & \\
\hline Diabetes mellitus & $1428(27.6 \%)$ & & $476(27.6 \%)$ & & \\
\hline Hypertension & $4263(82.5 \%)$ & & $1421(82.5 \%)$ & & \\
\hline ECl score & $9.81 \pm 4.23$ & & $9.81 \pm 4.23$ & & 0.99 \\
\hline Complications w/in 2 yrs postop & & Control & Osteopenia & Osteoporosis & \\
\hline Pseudarthrosis & $321(6.2 \%)$ & $118(6.8 \%)$ & $132(7.7 \%)$ & $71(4.1 \%)$ & $<0.05$ \\
\hline Revision surgery & $276(5.3 \%)$ & $108(6.3 \%)$ & $127(7.4 \%)$ & $41(2.4 \%)$ & $<0.05$ \\
\hline
\end{tabular}

Values are presented as number of patients (\%) unless otherwise indicated. Mean values are presented \pm SD. Patient groups were 1:1 propensity matched for baseline demographic characteristics and comorbidities.

\section{Effects of Pretreatment of Osteoporosis and Osteopenia on Postoperative Pseudarthrosis and Revision Surgery}

Patients with osteopenia who were not pretreated with antiosteoporotic medications prior to surgery had higher rates of pseudarthrosis $(6.38 \%$ vs $0.52 \%, p=0.306)$ and need for revision surgery $(5.51 \%$ vs $0.75 \%, \mathrm{p}=0.554)$ than patients with osteopenia who were pretreated prior to surgery, although this difference was not statistically significant (Tables 2 and 3, Fig. 3). Similarly, patients with osteoporosis who were not pretreated prior to surgery had higher rates of pseudarthrosis (5.63\% vs $2.03 \%, \mathrm{p}=0.915)$ and need for revision surgery $(5.40 \%$ vs $1.97 \%, \mathrm{p}=0.973$ ) than pretreated osteoporotic patients, although this difference was also not statistically significant (Tables 2 and 3, Fig. 4).

TABLE 2. Patient bone loss prevention medications taken $\geq 30$ days preoperatively

\begin{tabular}{lccc}
\hline & Osteopenia & Osteoporosis & p Value \\
\hline Any medication & $172(10.0 \%)$ & $477(27.7 \%)$ & $<0.05$ \\
\hline Teriparatide & $2(1.2 \%)$ & $18(3.8 \%)$ & $<0.05$ \\
\hline Bisphosphonates & $170(98.8 \%)$ & $453(95.0 \%)$ & $<0.05$ \\
\hline Denosumab & $0(0 \%)$ & $6(1.3 \%)$ & 0.073 \\
\hline
\end{tabular}

Values are presented as number of patients (\%) unless otherwise indicated.
Furthermore, the odds of pseudarthrosis and need for revision surgery in patients with osteopenia or osteoporosis were not significantly different between patients pretreated prior to surgery and those not treated prior to surgery (pseudarthrosis: OR $0.643,95 \%$ CI $0.308-1.343$ and OR $0.958,95 \%$ CI $0.641-1.432$; revision surgery: OR $1.262,95 \%$ CI $0.691-2.305$ and OR $0.972,95 \%$ CI $0.646-1.461$, with osteopenia or osteoporosis, respectively; Table 5).

\section{Discussion}

Osteoporosis and osteopenia are common among older adults with degenerative disorders of the spine. Patients with osteoporosis or osteopenia undergoing lumbar fusions are at an elevated risk for a number of postoperative complications that may require revision surgeryincluding pedicle screw loosening, nonunion, proximal junctional kyphosis, and adjacent segment fractures, ${ }^{5,8-11}$ The primary aim of this study was to investigate the effects of osteopenia or osteoporosis on the development of pseudarthrosis and need for revision surgery following single-level lumbar spinal fusion. The secondary aim was to compare these same outcome measures, stratifying by the preoperative use of disease-modifying drugs (e.g., teriparatide, bisphosphonates, and denosumab) that prevent bone loss. To accomplish these aims, 1:1 propensity score matching was used to create 3 equal cohorts of 
TABLE 3. Complications occurring $\leq 2$ years postoperatively in patients taking and those not taking bone loss prevention medication

\begin{tabular}{crrrrrrrr}
\hline & \multicolumn{3}{c}{ Osteopenia } & & \multicolumn{3}{c}{ Osteoporosis } \\
\cline { 2 - 3 } Complication & w/ Meds & w/o Meds & p Value & & w/ Meds & w/o Meds & p Value \\
\hline Pseudarthrosis & $9(0.5 \%)$ & $110(6.4 \%)$ & 0.306 & & $35(2.0 \%)$ & $97(5.6 \%)$ & 0.915 \\
\hline Revision surgery & $13(0.8 \%)$ & $95(5.5 \%)$ & 0.554 & & $34(2.0 \%)$ & $93(5.4 \%)$ & 0.973 \\
\hline
\end{tabular}

Values are presented as number of patients (\%) unless otherwise indicated.

1723 patients (normal BMD, osteopenia, and osteoporosis) with similar baseline demographic and comorbidity profiles. The odds of pseudarthrosis and need for revision surgery in patients with osteopenia or osteoporosis were 2-fold to 3-fold higher than those in patients in the control group (normal BMD). In a subgroup analysis, the odds of pseudarthrosis in patients with osteoporosis did not differ from those in patients with osteopenia, suggesting that for single-level fusion there may be limited additional risk for patients with osteoporosis versus osteopenia.

The majority of patients with either osteoporosis or osteopenia were not using medications that could alter these disease states; of note, however, medications were more frequently used in patients with osteoporosis $(27.7 \%$ vs $10.0 \%$ ). Among those patients using medications, the most commonly used agents by far were bisphosphonates (98.8\% of osteopenia patients and $95.0 \%$ of osteoporosis patients). These results may indicate a general underutilization of medications that could alter BMD, in addition to specific underutilization of newer agents such as teriparatide and denosumab. While pretreated patients with either osteoporosis or osteopenia had lower rates of pseudarthrosis and revision surgery, these differences were not found to be statistically significant. These results suggest unclear benefits from pretreatment in regard to the risk of pseudarthrosis and need for revision surgery in patients undergoing a single-level lumbar fusion. Conceivably, the benefit of pretreatment may be more pronounced in patients requiring longer fusion constructs.

While the current literature is limited in regard to inves- tigations pertaining to the association of BMD and rates of pseudarthrosis and need for revision surgery, several studies and surveys merit mention. Highlighting the modest awareness of these factors during workup, a survey of 93 spine surgeons found that, prior to instrumented fusion, only $44 \%$ of surgeons assessed for osteoporosis using a DEXA (dual-energy x-ray absorptiometry) scan, and only $12 \%$ assessed for this state with metabolic laboratory analysis. Rates of osteoporosis workup were even lower prior to the treatment of pseudarthrosis (22\% and $11 \%$, respectively). ${ }^{12}$ Similar results were found in a survey by DíazRomero Paz et al. of 77 spine surgeons, which indicated that $32.5 \%$ of surgeons did not perform any workup prior to fusion in patients with suspected osteoporosis, and $37.7 \%$ of surgeons would proceed to surgery without any treatment. ${ }^{8}$ Both of these surveys underscore that a significant portion of spine surgeons may see limited benefit in osteoporotic workups prior to surgery; however, these patterns of care may change significantly with further evidence that poor BMD is associated with poor surgical outcomes but is treatable with increasingly effective strategies.

In a series of 140 patients, Bjerke et al. observed that patients with $\mathrm{T}$ scores below -1.0 had a greater risk for osteoporosis-related complications (revision surgery), compression fracture, proximal junctional kyphosis, pseudarthrosis, and failure of instrumentation. Similar to the findings of our population-level analysis on pseudarthrosis and need for revision surgery, both osteopenia and osteoporosis were found to significantly impact the aforementioned surgical outcomes? 7 A number of previ-
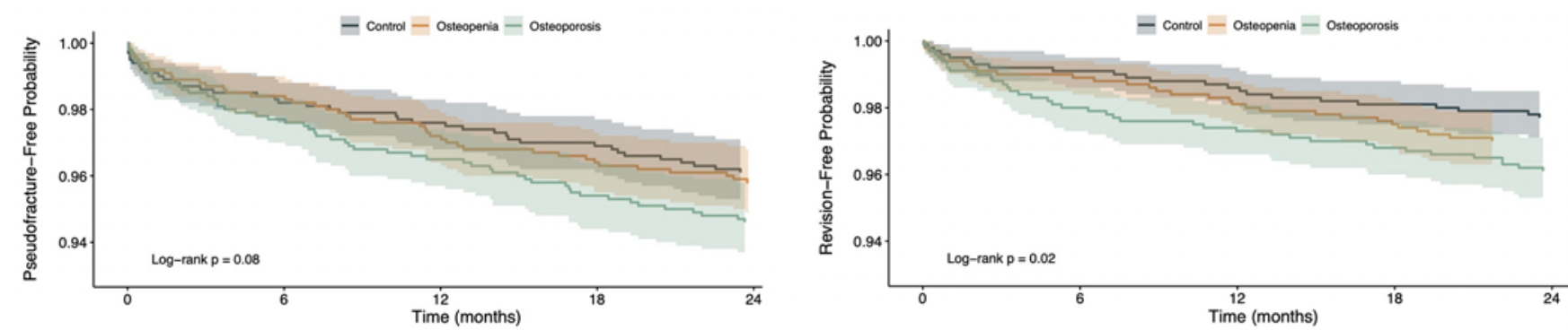

$\begin{array}{ccccc}\text { Number at risk } & & & & \\ 1723 & 1692 & 1681 & 1670 & 0 \\ 1723 & 1696 & 1674 & 1661 & 0 \\ 1723 & 1683 & 1663 & 1643 & 0 \\ \text { Cumulative number of events } & & & \\ 0 & 31 & 42 & 53 & 67 \\ 0 & 27 & 49 & 62 & 73 \\ 0 & 40 & 60 & 80 & 93\end{array}$

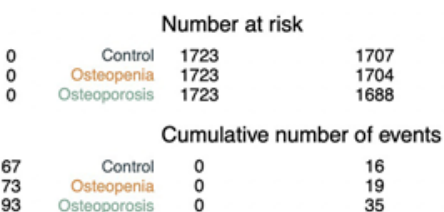

\begin{tabular}{ll}
1699 & \\
1691 & 169 \\
1677 & 16 \\
& 16 \\
& \\
24 & \\
32 & 32 \\
46 & 53 \\
\hline
\end{tabular}

FIG. 2. Kaplan-Meier plot for pseudarthrosis and revision surgery occurring within 2 years postoperatively. 
TABLE 4. Complications $\leq 2$ years postoperatively in patients compared with controls

\begin{tabular}{ccc}
\hline \multicolumn{1}{c}{ Complication } & Osteopenia & Osteoporosis \\
\hline Pseudarthrosis & $1.7(1.26-2.30)$ & $1.92(1.43-2.59)$ \\
\hline Revision surgery & $2.73(1.89-3.94)$ & $3.25(2.27-4.65)$ \\
\hline
\end{tabular}

Values are presented as OR $(95 \% \mathrm{Cl})$.

ously reported investigations have indicated the possible benefits of agents such as bisphosphonates and teriparatide in osteoporotic patients undergoing spinal fusion., 913,14 Buerba et al. reported a recent meta-analysis in which, while no statistically significant difference was noted between bisphosphonates and control groups regarding fusion rate $(\mathrm{OR} 2.2,95 \% \mathrm{CI} 0.87-5.56, \mathrm{p}=0.09)$, teriparatide was associated with higher fusion rates than bisphosphonates (OR 2.3, 95\% CI 1.55-4.32, p < 0.0001). ${ }^{15}$ While our analysis was not adequately powered to assess the specific effects of teriparatide, we similarly found no statistically significant difference in rates of pseudarthrosis or need for revision surgery in pretreated osteoporotic patients.
However, the lower rates of pseudarthrosis and need for revision surgery in both the osteoporosis and osteopenia groups in the current study warrant further investigation with larger prospective studies.

\section{Study Limitations}

There are a number of limitations that should be considered when interpreting the results of this study. Our study relied on ICD-9-CM and ICD-10-CM codes for identification of patient demographic and outcome characteristics; hence, underreporting, missed codes, and/or inaccurate codes were possible and may have affected our results. While we assume that coders and providers will generally defer to $\mathrm{T}$ scores found in DEXA scans for appropriate diagnosis of osteopenia and osteoporosis, we were unable to specifically extract these elements from DEXA reports. Exactly how the presence of pseudarthrosis was determined by providers is unclear, and we concede that this is a significant limitation in our analysis. Patients coded as having pseudarthrosis were most likely symptomatic; thus, the true incidence proportion of all cases of nonunion (symptomatic and asymptomatic) may be underestimated in our analysis. Furthermore, whether pseudarthrosis was
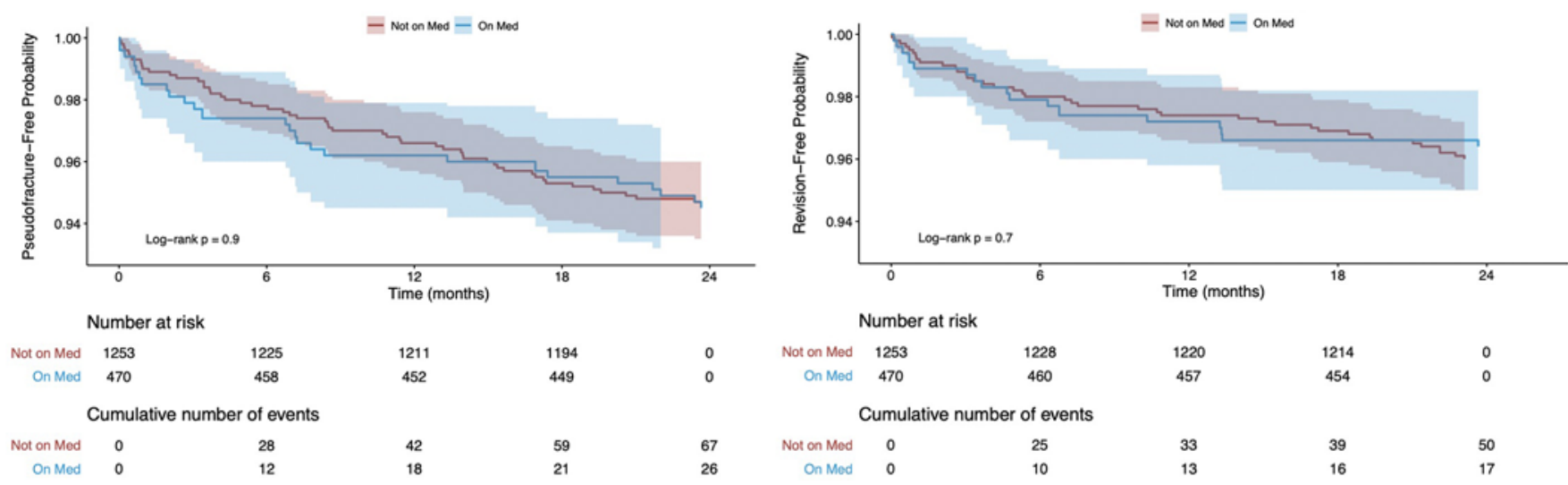

FIG. 3. Kaplan-Meier plot for pseudarthrosis and revision surgery occurring within 2 years postoperatively in osteopenia patients with and without medications (Med) taken within 30 days preoperatively.
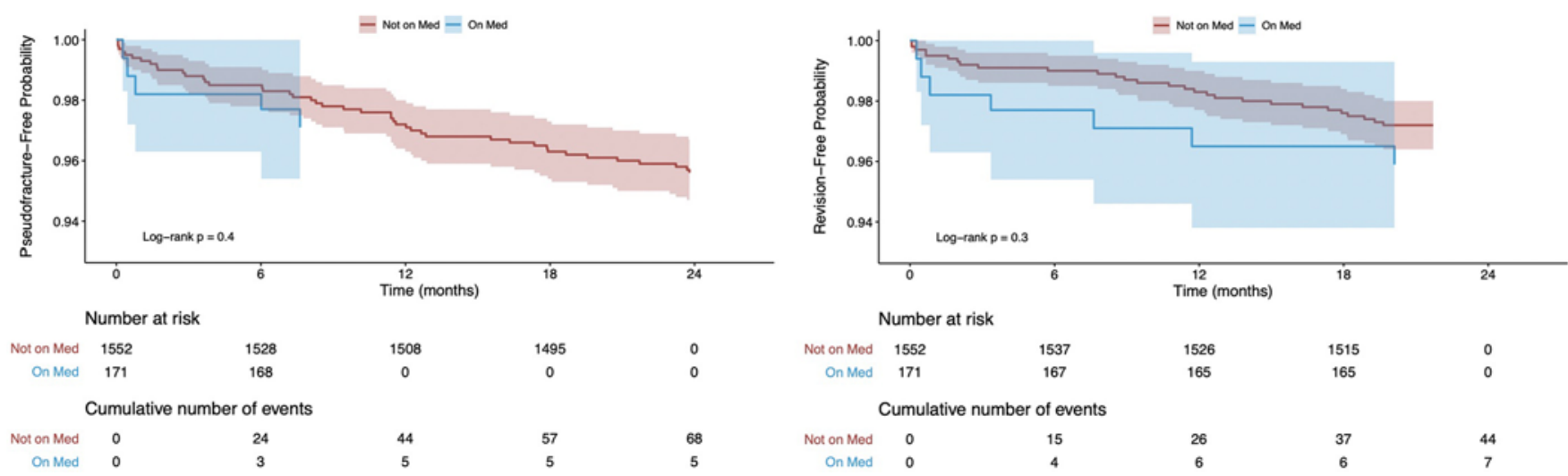

FIG. 4. Kaplan-Meier plot for pseudarthrosis and revision surgery occurring within 2 years postoperatively in osteoporosis patients with and without medications taken within 30 days preoperatively. 
TABLE 5. Complications $\leq 2$ years postoperatively in patients in the same cohort taking compared with those not taking bone loss prevention medication $\geq \mathbf{3 0}$ days preoperatively

\begin{tabular}{lcc}
\hline Complication & $\begin{array}{c}\text { Osteopenia on } \\
\text { Any Medication }\end{array}$ & $\begin{array}{c}\text { Osteoporosis on } \\
\text { Any Medication }\end{array}$ \\
\hline Pseudarthrosis & $0.643(0.308-1.343)$ & $0.958(0.641-1.432)$ \\
\hline Revision surgery & $1.262(0.691-2.305)$ & $0.972(0.646-1.461)$ \\
\hline
\end{tabular}

Values are presented as $\mathrm{OR}(95 \% \mathrm{Cl})$.

the causative factor in subsequent revision surgery was unclear. Regarding medication usage, we did not stratify by dosage of agents, which may have significantly altered our results. In addition, patients receiving antiosteoporotic medications may be those at the highest risk, contributing to the risk of selection bias. Although only those patients who had an active medical record and were insured by $\mathrm{Hu}-$ mana for a period of 2 years were included, the absolute interval of postoperative follow-up that patients spent with their individual surgeon is not known. In addition, we only queried patients who received preoperative medication, and further investigation may be warranted to assess the impact of these medications if used in the postoperative period.

Despite these limitations, we hope that surgeons find our analysis both interesting and clinically relevant to their daily practice. Perhaps most importantly, we hope that clinicians may be more inclined to perform comprehensive preoperative evaluations for patients with poor BMD, with the knowledge that both osteopenia and osteoporosis significantly impact surgical outcomes. In addition, to prevent loss of BMD, the use of medications, even though the evidence for their effectiveness is unclear, may be a worthwhile consideration in high-risk patients. Finally, we hope that these findings generate interest that may lead to further investigations into osteoporotic patients, potential treatment regimens, and spinal surgery outcomes.

\section{Conclusions}

Postoperative pseudarthrosis and revision surgery rates following single-level lumbar spinal fusion are significantly higher in patients with osteopenia and osteoporosis than in patients with normal BMD. Pretreatment with medications to prevent bone loss prior to surgery decreased these complication rates, although the observed differences did not reach statistical significance.

\section{References}

1. Pannell WC, Savin DD, Scott TP, et al. Trends in the surgical treatment of lumbar spine disease in the United States. Spine J. 2015;15(8):1719-1727.

2. Rushton A, Staal JB, Verra M, et al. Patient journey following lumbar spinal fusion surgery (LSFS): protocol for a multicentre qualitative analysis of the patient rehabilitation experience (FuJourn). BMJ Open. 2018;8(1):e020710.

3. Lubelski D, Choma TJ, Steinmetz MP, et al. Perioperative medical management of spine surgery patients with osteoporosis. Neurosurgery. 2015;77(suppl 4):S92-S97.

4. Tomé-Bermejo F, Piñera AR, Alvarez-Galovich L. Osteoporosis and the management of spinal degenerative disease (I). Arch Bone Jt Surg. 2017;5(5):272-282.
5. Park SB, Chung CK. Strategies of spinal fusion on osteoporotic spine. J Korean Neurosurg Soc. 2011;49(6):317-322.

6. Khosla S, Melton LJ III. Clinical practice. Osteopenia. $N$ Engl J Med. 2007;356(22):2293-2300.

7. Bjerke BT, Zarrabian M, Aleem IS, et al. Incidence of osteoporosis-related complications following posterior lumbar fusion. Global Spine J. 2018;8(6):563-569.

8. Díaz-Romero Paz R, Sosa Henríquez M, Armas Melián K, Coloma Valverde G. Trends and attitudes of spine surgeons regarding osteoporosis. Article in Spanish. Neurocirugia (Astur). 2019;30(6):268-277.

9. Morris MT, Tarpada SP, Tabatabaie V, Cho W. Medical optimization of lumbar fusion in the osteoporotic patient. Arch Osteoporos. 2018;13(1):26.

10. Uei H, Tokuhashi Y, Maseda M, et al. Exploratory analysis of predictors of revision surgery for proximal junctional kyphosis or additional postoperative vertebral fracture following adult spinal deformity surgery in elderly patients: a retrospective cohort study. J Orthop Surg Res. 2018;13(1):252.

11. Chin DK, Park JY, Yoon YS, et al. Prevalence of osteoporosis in patients requiring spine surgery: incidence and significance of osteoporosis in spine disease. Osteoporos Int. 2007; 18(9):1219-1224.

12. Rittweger J. Can exercise prevent osteoporosis? J Musculoskelet Neuronal Interact. 2006;6(2):162-166.

13. Chaudhary N, Lee JS, Wu JY, Tharin S. Evidence for use of teriparatide in spinal fusion surgery in osteoporotic patients. World Neurosurg. 2017;100:551-556.

14. Hassanzadeh H, Puvanesarajah V, Dalkin AC. Medical management of osteoporosis for elective spine surgery. Clin Spine Surg. 2016;29(4):134-140.

15. Buerba RA, Sharma A, Ziino C, et al. Bisphosphonate and teriparatide use in thoracolumbar spinal fusion: a systematic review and meta-analysis of comparative studies. Spine (Phila Pa 1976). 2018;43(17):E1014-E1023.

\section{Disclosures}

The authors report no conflict of interest concerning the materials or methods used in this study or the findings specified in this paper.

\section{Author Contributions}

Conception and design: Adogwa, Khalid. Acquisition of data: Khalid, Maasarani. Analysis and interpretation of data: Adogwa, Khalid, Maasarani. Drafting the article: Adogwa, Khalid, Nunna, Maasarani, Belmont. Critically revising the article: Adogwa, Khalid, Nunna, Maasarani, Deme, Chilakapati, Eldridge, Singh, Bagley. Reviewed submitted version of manuscript: all authors. Approved the final version of the manuscript on behalf of all authors: Adogwa. Statistical analysis: Adogwa, Khalid, Nunna, Maasarani. Administrative/technical/material support: Adogwa, Khalid, Nunna, Deme, Chilakapati, Eldridge, Singh, Bagley. Study supervision: Adogwa, Khalid.

\section{Supplemental Information \\ Videos}

Video Abstract. https://vimeo.com/438530966.

\section{Online-Only Content}

Supplemental material is available online.

Supplemental Tables 1-6. https://thejns.org/doi/suppl/10.3171/ 2020.5.FOCUS20289.

\section{Correspondence}

Owoicho Adogwa: University of Texas Southwestern Medical School, Dallas, TX. owoicho.adogwa@gmail.com. 